\title{
A patient with acute macular neuroretinopathy and central retinal vein occlusion
}

This article was published in the following Dove Press journal:

Clinical Ophthalmology

12 July 2013

Number of times this article has been viewed

\author{
Kiriko Hirooka' \\ Wataru Saito ${ }^{1,2}$ \\ Kousuke Noda ${ }^{1,2}$ \\ Susumu Ishida ${ }^{1,2}$ \\ 'Department of Ophthalmology, \\ Hokkaido University Graduate \\ School of Medicine, Sapporo, Japan; \\ ${ }^{2}$ Department of Ocular Circulation \\ and Metabolism, Hokkaido University \\ Graduate School of Medicine, \\ Sapporo, Japan
}

Purpose: The precise mechanism causing acute macular neuroretinopathy (AMN) is still unknown. A recent report suggested that choroidal circulation impairment correlates with its pathogenesis. We report a rare case with simultaneous onset of AMN and central retinal vein occlusion (CRVO), which is a retinal circulation disorder.

Methods: Case report.

Results: A 44-year-old woman complained of central visual loss of the left eye for the previous 2 weeks. The patient's visual acuity was 0.5 in the left eye (OS). Fundoscopic examination revealed a wedge-shaped, dark reddish-brown lesion at the macula, and CRVO-like retinal hemorrhages OS. Fluorescein angiography revealed retinal vasculitis and hypofluorescence corresponding to the macular lesion. The patient's scanning laser ophthalmoscopy infrared imaging result led to a diagnosis of AMN. Two weeks after corticosteroid pulse therapy, her visual acuity improved to 1.2 OS, with improvement of macular findings and Humphrey perimetry. When the dose of oral corticosteroid was decreased, the AMN lesion worsened, with recurrence of retinal hemorrhages. Visual functions improved again after an increased dose of corticosteroid.

Conclusion: These results suggest that circulatory disorders almost simultaneously occurred in choroidal and retinal vessels, resulting in the onset of both AMN and CRVO.

Keywords: choroidal circulation, optical coherence tomography, retinal circulation, systemic corticosteroid therapy

\section{Introduction}

Acute macular neuroretinopathy $(\mathrm{AMN})$ is a rare disease characterized by a wedgeshaped, dark reddish-brown lesion at the macula. ${ }^{1}$ Scanning laser ophthalmoscopy (SLO) infrared imaging clearly reveals these lesions as hyporeflectivity, which is a characteristic finding in AMN, and this abnormal finding is present even though AMN lesions are not biomicroscopically evident. ${ }^{2}$ The development of spectral-domain optical coherence tomography (OCT) demonstrated that AMN lesions mainly affect the outer retina and not the inner retina. ${ }^{2}$ Recently, it was reported that hyper-reflective lesions at the level of the outer plexiform and outer nuclear layers on OCT temporally appeared despite an intact photoreceptor inner/outer segment junction (IS/OS) in patients with early-stage AMN; thereafter, the IS/OS was affected at the same sites. ${ }^{3}$ Ischemia in the deep inner retinal capillary vessels was speculated as the cause of these newly discovered early OCT lesions, ${ }^{3}$ because retinal circulation provides photoreceptors with $10 \%$ of their oxygen supply. These results suggest the onset of both inner and outer retinal disorders in AMN.

Approximately $10 \%$ of AMN patients appear to be complicated by intraretinal hemorrhages, which are inner retinal disorders. ${ }^{4,5}$ However, the relationship between
Correspondence: Wataru Saito Department of Ocular Circulation and Metabolism, Hokkaido University Graduate School of Medicine, Nishi 7, Kita I5, Kita-ku, Sapporo 060-8638, Japan Tel +8I I | 7065944

Fax +8 I II 7065948

Email wsaito@med.hokudai.ac.jp 
these conditions is unknown. Herein, we describe the case of a patient who developed AMN and central retinal vein occlusion (CRVO).

\section{Case report}

A 44-year-old woman noticed central visual loss with photopsia in her left eye for the previous 2 weeks. The patient's medical and family histories were unremarkable; however, she took oral contraceptives for menstrual irregularities.

The patient's best-corrected visual acuity (BCVA) was 1.2 in the right eye (OD) and 0.5 in the left eye (OS). There were no abnormal ocular findings OD. Slit-lamp examination revealed mild anterior vitreous cells OS. Fundoscopic examination revealed a wedge-shaped, dark reddish-brown lesion at the macula, brush- or blot-like intraretinal hemorrhages that spread in a pandirectional manner from the optic disc, a mildly swollen optic disc, and dilatation and tortuosity of the retinal veins (Figure 1A). SLO infrared imaging revealed that the dark area corresponded to the dark reddish lesion (Figure 1B). Fluorescein angiography (FA) revealed hypofluorescence corresponding to the macular lesion and retinal hemorrhages in the initial phase (Figure $1 \mathrm{C}$ ), and retinal phlebitis and the leakages from the optic disc in the late phase (Figure 1D). Indocyanine green angiography (ICGA) revealed a normal appearance during the initial phase, and spotted hypofluorescence at the macular area during the late phase (Figure 1E). Humphrey threshold 10-2 perimetry showed a central scotoma (mean deviation [MD] value: $-9.40 \mathrm{~dB}$ ) corresponding to the lesion area (Figure 1F). Goldmann perimetry showed a central scotoma with enlargement of the blind spot. Multifocal electroretinography revealed decreased amplitude on the lesion area. OCT showed loss of the photoreceptor IS/OS corresponding to the dark reddish lesion (Figure 1G). No macular edema was evident, possibly because the relatively mild occlusion of retinal veins appeared to be restricted mainly to the superotemporal branch and two nasal branches. Systemic screening comprising serological analysis or antibody titres for infectious diseases, including syphilis; QuantiFERON; serum autoantibodies, including antiphospholipid antibody; full blood counts; erythrocyte sedimentation rate; serum lipids; serum and urine glucose; hemoglobin $\mathrm{A}_{1 \mathrm{c}}$; and endogenous coagulation function revealed no abnormalities in the patient. The patient was diagnosed with AMN and CRVO OS and was followed up with withdrawal of oral contraceptives and no medication.

Two months after the first visit, the area of the macular lesion and BCVA remained almost unchanged, although most of the retinal hemorrhages spontaneously disappeared

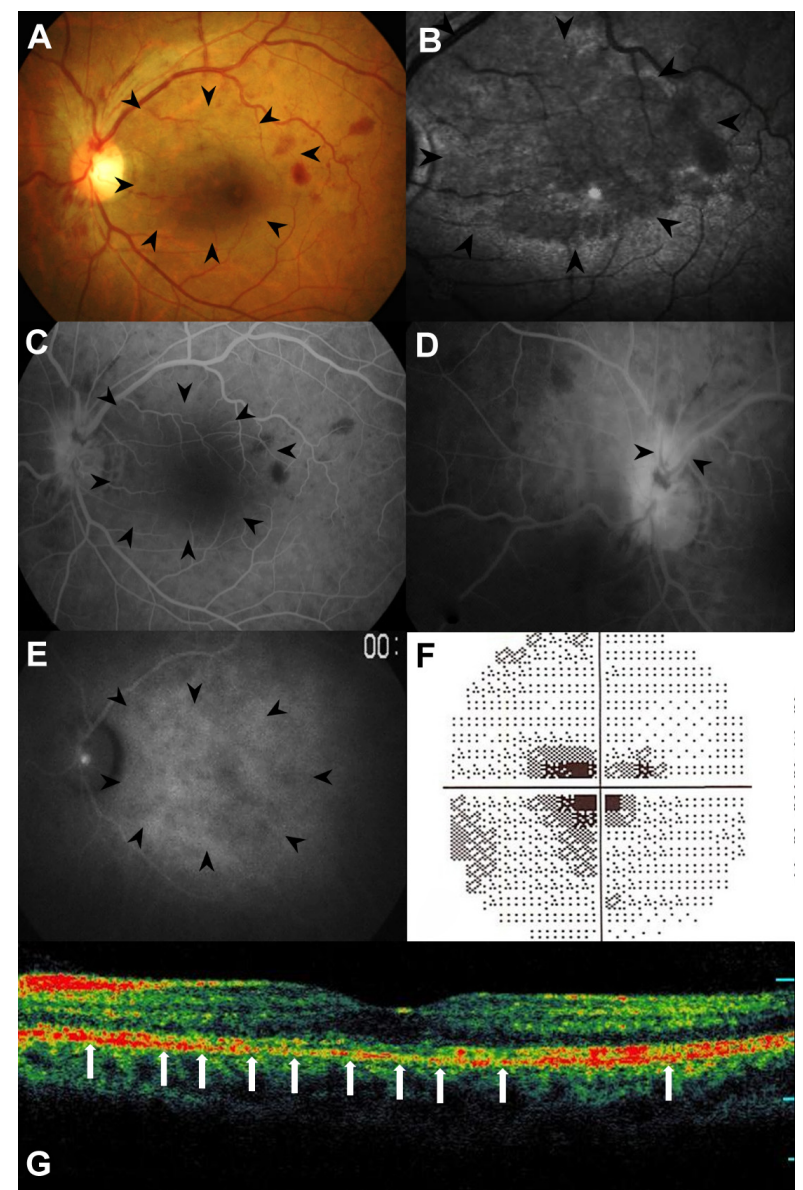

Figure I Findings in the left eye at the first visit: (A) the fundus photograph showed a wedge-shaped, dark reddish lesion at the macula (arrowheads), brush- or blotlike intraretinal hemorrhages that spread in a pandirectional manner from the optic disc, a mildly swollen optic disc, and dilated and tortuous retinal veins; (B) scanning laser ophthalmoscopy infrared imaging showed the dark area corresponding to the dark reddish lesion (arrowheads); (C and D) fluorescein angiography revealed hypofluorescence corresponding to the macular lesion (arrowheads) and retinal hemorrhages at the initial phase $(\mathbf{C})$ and retinal phlebitis (arrowheads) and leakages from the optic disc in the late phase (D); (E) on indocyanine green angiography, the dark reddish lesion showed spotted hypofluorescence during the late phase (arrowheads); (F) Humphrey threshold 10-2 perimetry revealed a central scotoma (mean deviation value: $-9.40 \mathrm{~dB}$ ); (G) optical coherence tomography showed the loss of the photoreceptor inner/outer segment junction corresponding to the macular lesion (arrows).

(Figure 2A), together with the resolution of retinal phlebitis and the leakages from the optic disc on late-phase FA (Figure 2B). After informed consent was obtained, corticosteroids were initially administered as per the following schedule: intravenous methyl-prednisolone (mPSL) at $1,000 \mathrm{mg} /$ day for 3 days, oral PSL at $30 \mathrm{mg} /$ day for 7 days, and intravenous mPSL at $1,000 \mathrm{mg}$ /day for 3 days. Oral PSL (30 mg/day) was then administrated and tapered by $10 \mathrm{mg}$ /day every 1 month. The patient's BCVA improved to 1.2 OS 2 weeks after treatment. The dark reddish-brown lesion had shrunk (Figure 2C), and the SLO dark area was reduced (Figure 2D). Humphrey perimetry showed that the central scotoma had shrunk, with marked improvement of 


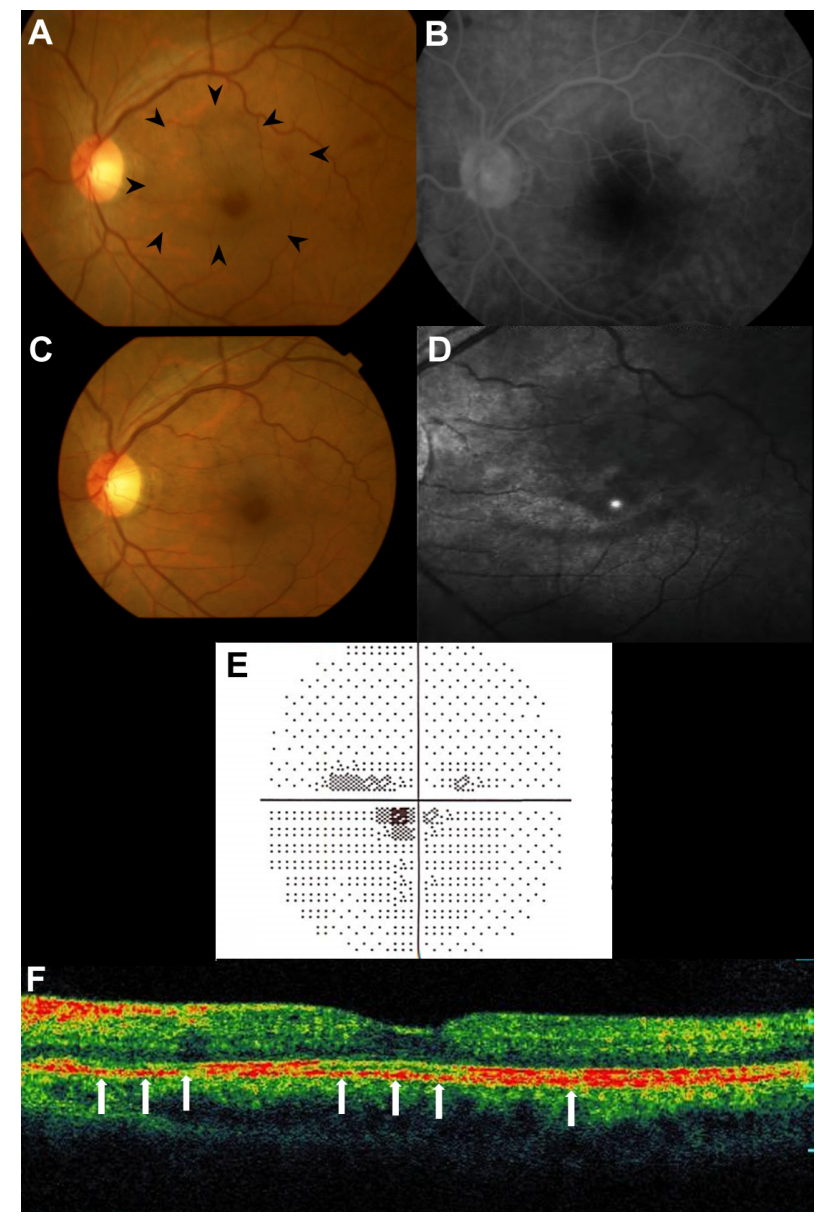

Figure 2 Photographs of the left eye 2 months after the first visit ( $\mathbf{A}$ and $\mathbf{B}$ ) and 2 weeks after corticosteroid pulse therapy (C-F): (A) fundus photograph showing the spontaneous absorption of the retinal hemorrhages, although the area of the macular lesion remained unchanged (arrowheads); (B) on late-phase fluorescein angiography, retinal phlebitis and the leakages from the optic disc resolved; (C) the dark reddish lesion improved; (D) the dark area on scanning laser ophthalmoscopy at the lesion site was reduced; (E) on perimetry, the central scotoma had shrunk (mean deviation value: $-3.58 \mathrm{~dB}$ ); (F) on optical coherence tomography, the discontinuity of the photoreceptor inner/outer segment junction (arrows) had improved at the fovea.

MD value ( $-3.58 \mathrm{~dB})$ (Figure $2 \mathrm{E})$, and that the discontinuity of the IS/OS at the macula had improved (Figure 2F).

Three months after treatment (during oral PSL administration at $15 \mathrm{mg} /$ day), her BCVA was reduced to $0.6 \mathrm{OS}$, the macular lesion and MD value $(-7.42 \mathrm{~dB})$ worsened, and the intraretinal hemorrhages recurred. The patient received an increased oral PSL dose (30 mg/day) and a posterior subTenon injection of triamcinolone acetonide $(40 \mathrm{mg}) \mathrm{OS}$. The AMN lesion again improved, with the disappearance of the retinal hemorrhages, and BCVA and MD values increased to 0.9 and $-6.08 \mathrm{~dB}$, respectively, 6 months after the initial treatment. Thereafter, PSL was tapered by $5 \mathrm{mg}$ /day every 2-3 months and was continued for 18 months.

Sixty months after the first visit, the patient's BCVA and MD values were 0.8 and $-3.88 \mathrm{~dB}$, respectively. The dark reddish lesion was faintly observed without a dark area on SLO infrared imaging (Figure 3A and B). Late-phase FA and ICGA showed resolution of the hypofluorescent lesions at the macula (Figure 3C and D). OCT revealed complete recovery of the IS/OS, with partial discontinuity of the cone outer segment tip line (Figure 3E, arrows).

\section{Discussion}

In this study, we describe an AMN patient with nonischemic CRVO. The lesions and visual functions improved at an early stage after systemic corticosteroid therapy. To our knowledge, this is the first reported AMN case with CRVO.

AMN with intraretinal hemorrhages was previously reported in six patients, ${ }^{4,5}$ however, the extent of retinal hemorrhage was mild in those cases. Retinal capillary disorders following the incidental complication of systemic hypertension was inferred as the cause of intraretinal hemorrhages associated with AMN. ${ }^{4,5}$ However, our patient had no history of arteriosclerotic diseases, including hypertension.

AMN lesions can show hypofluorescence on FA and ICGA, ${ }^{6}$ as observed in the present case. We recently used laser speckle flowgraphy to demonstrate reduced choroidal blood flow velocity at the lesion site during the acute phase

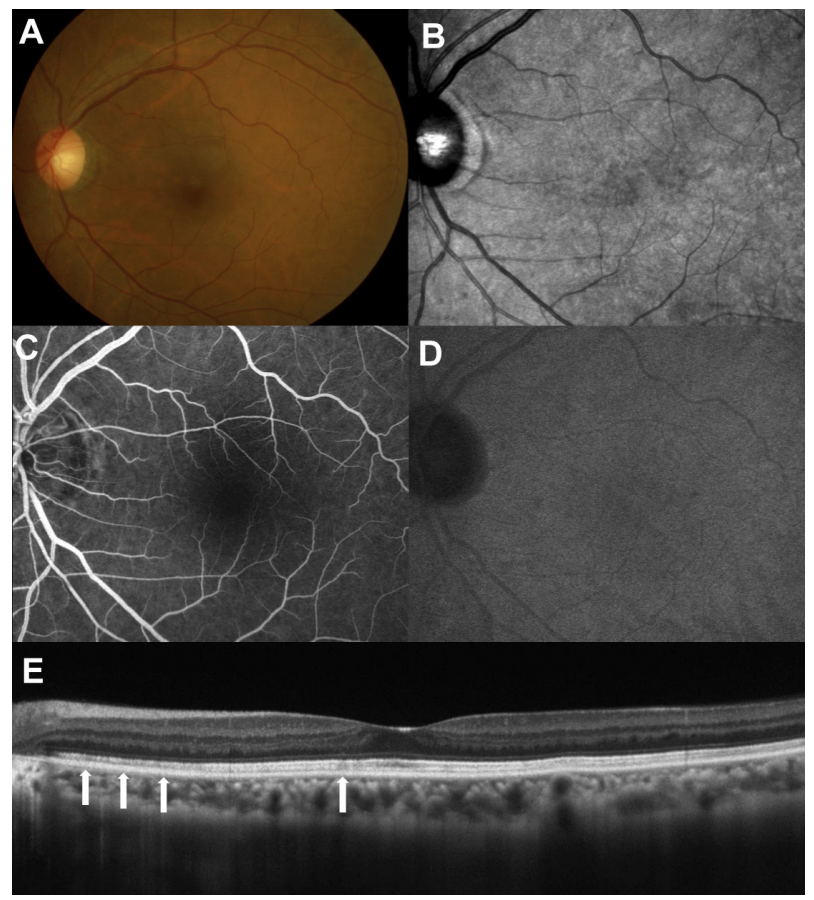

Figure 3 Findings in the left eye 60 months after the first visit: (A and B) the dark reddish lesion (A) was faintly observed without a dark area on scanning laser ophthalmoscopy infrared imaging (B); (C and D) on late-phase fluorescein angiography (C) and indocyanine green angiography (D), hypofluorescent lesions corresponding to the acute macular neuroretinopathy lesion almost resolved; (E) optical coherence tomography revealed complete recovery of the inner/outer segment junction with partial discontinuity of the cone outer segment tip line (arrows). 
of AMN. ${ }^{6}$ In the present case, we could not evaluate choroidal circulation impairment using this technique, because of the patient's poor fixation. Nevertheless, the hypofluorescent areas observed on FA and ICGA at the lesion site suggest choroidal hypoperfusion in this case, because there were no obvious findings on OCT responsible for blocked fluorescence within the AMN lesion. These observations suggest that choroidal circulation impairment is related to the pathogenesis of AMN. On the other hand, CRVO is caused by circulation disturbances in retinal veins following the stenosis or occlusion of the central retinal vein near the lamina cribrosa from unknown causes. CRVO is associated with diseases causing arteriosclerosis in the elderly, whereas it is thought to be associated with vasculitis in young adults. ${ }^{7,8}$ When an AMN lesion recurred, retinal hemorrhages also appeared again. Therefore, we infer that the onset of both AMN and CRVO in the present case was due to circulatory disturbances that occurred almost simultaneously in both choroidal and retinal vessels. Inflammatory mechanisms are speculated because the patient had CRVO with retinal vasculitis and because the functional and morphological abnormalities improved from the early stage after systemic corticosteroid administration, similar to findings in our recently reported case. ${ }^{6}$ However, thrombosis following oral contraceptive use $^{9,10}$ or other causes cannot be excluded, because in a large case series of 588 patients with retinal vein occlusion, six of nine patients aged younger than 35 years were taking oral contraceptive pills. ${ }^{10}$ Further studies regarding retinal and choroidal blood flow are needed in AMN patients.

In the literature review, AMN lesions showed no spontaneous improvement in $65 \%$ of patients. ${ }^{11}$ At present, therapeutic strategies for AMN remain to be established. In this case, the IS/OS improved from the early stage after systemic corticosteroid therapy, and finally completely recovered, as in our recently reported case. ${ }^{6}$ Thus, these results suggest the potential efficacy of systemic corticosteroid therapy for some AMN patients, and warrant future prospective studies to examine this intervention.

\section{Conclusion}

We reported a case of AMN with CRVO. The results suggest that circulatory disorders in the retina and/or choroid relate to the etiology of AMN.

\section{Disclosure}

The authors report no conflicts of interest in relation to this work.

\section{References}

1. Bos PJ, Deutman AF. Acute macular neuroretinopathy. Am J Ophthalmol. 1975;80(4):573-584.

2. Neuhann IM, Inhoffen W, Koerner S, Bartz-Schmidt KU, Gelisken F. Visualization and follow-up of acute macular neuroretinopathy with the Spectralis ${ }^{\circledR}$ HRA+OCT device. Grafes Arch Clin Exp Ophthalmol. 2010;248(7):1041-1044.

3. Fawzi AA, Pappuru RR, Sarraf D, et al. Acute macular neurortinopathy: long-term insights revealed by multimodalimaging. Retina. 2012;32(8):1500-1513.

4. Priluck IA, Buettner H, Robertson DM. Acute macular neuroretinopathy. Am J Ophthalmol. 1978;86(6):775-778.

5. Gillies M, Sarks J, Dunlop C, Mitchell P. Traumatic retinopathy resembling acute macular neuroretinopathy. Aust $N$ Z J Ophthalmol. 1997;25(3):207-210.

6. Hashimoto Y, Saito W, Mori S, Saito M, Ishida S. Increased macular choroidal blood flow velocity during systemic corticosteroid therapy in a patient with acute macular neuroretinopathy. Clin Ophthalmol. 2012;6:1645-1649.

7. Yoshizawa C, Saito W, Kase M, Ishida S. Clinical features of central retinal vein occlusion with inflammatory etiology. Asia Pac $J$ Ophthalmol. 2012;1(5):270-276.

8. The Eye Disease Control Study Group. Risk factor for central retinal vein occlusion. Arch Ophthalmol. 1996;114(5):545-554.

9. Van Hylckama Vlieg A, Helmerhorst FM, Vandenbroucke JP, Doggen CJ, Rosendaal FR. The venous thrombotic risk of oral contraceptives, effects of oestrogen dose and progestogen type: results of the MEGA case-control study. BMJ. 2009;339:b2921.

10. Kirwan JF, Tsaloumas MD, Vinall H, Prior P, Kritzinger EE, Dodson PM. Sex hormone preparations and retinal vein occlusion. Eye (Lond). 1997;11(Pt 1):53-56.

11. Turbeville SD, Cowan LD, Gass JDM. Acute macular neuroretinopathy: a review of the literature. Surv Ophthalmol. 2003;48(1):1-11.
Clinical Ophthalmology

\section{Publish your work in this journal}

Clinical Ophthalmology is an international, peer-reviewed journal covering all subspecialties within ophthalmology. Key topics include: Optometry; Visual science; Pharmacology and drug therapy in eye diseases; Basic Sciences; Primary and Secondary eye care; Patient Safety and Quality of Care Improvements. This journal is indexed on Submit your manuscript here: http://www.dovepress.com/clinical-ophthalmology-journal

\section{Dovepress}

PubMed Central and CAS, and is the official journal of The Society of Clinical Ophthalmology (SCO). The manuscript management system is completely online and includes a very quick and fair peer-review system, which is all easy to use. Visit http://www.dovepress.com/ testimonials.php to read real quotes from published authors. 